\title{
Reproducible Fabrication Method for Polarization Preserving Single-Mode Fiber Couplers
}

\author{
M. ABEBE, CARL A. VILLARRUEL, AND WILLIAM K. BURNS, MEMBER, IEEE
}

\begin{abstract}
The adaptation of the biconically-tapered-fused (BTF) fiber coupler fabrication approach to polarization preserving fiber couplers is reported. Two practical methods for identifying and aligning the birefringent axes of the fibers forming the coupler are described. The fabrication approaches are highly reproducible and the 3-dB couplers fabricated exhibit less than 1.0-dB insertion loss, controllable splitting ratio, and an average polarization extinction ratio of 15 to 20 dB.
\end{abstract}

\section{INTRODUCTION}

$S$ INGLE-MODE fiber couplers that preserve the state of polarization are critical components for applications where polarization noise is a problem, such as fiber gyroscopes, interferometric sensors, and coherent communications. To date, numerous laboratories have reported the performance, manufacturing method, and theory of operation for various types of polarization preserving fiber couplers [1]-[5]. In all cases, however, the quantities of devices involved were rather small, usually less than ten, and no reports of fabrication yield are available.

Initially, polarization preserving couplers were fabricated using ordinary polarization preserving fiber, either by the biconically-tapered-fused (BTF) [1], [2] or by polishing [3] techniques. The best results reported for 3-dB couplers fabricated using ordinary (circular outer diameter) polarization preserving fiber have been: 0.2 - to 5.0 $\mathrm{dB}$ insertion loss and 6- to $21-\mathrm{dB}$ polarization extinction. More recently, however, couplers have been fabricated by using polarization preserving fiber having a special geometrical feature, one or two flat facets on its outer surface, which are used to uniquely identify the orientation of the birefringent axes within the fiber. Both BTF [4] and fiber polishing [5] coupler fabrication techniques have worked well with these special fibers. The best results reported for 3-dB couplers fabricated with these special fibers have been: $0.1-$ to $3.0-\mathrm{dB}$ insertion loss and between 15- to 30-dB polarization extinction.

Here we report the development of a modified BTF manufacturing approach for high-performance polarization preserving couplers using stress-induced high birefringent fiber with a circular outer diameter. Results for

Manuscript received August 22, 1986; revised October 16, 1987

M. Abebe is with Sachs/Freeman Associates, Landover, MD 20785

C. A. Villarruel and W. K. Burns are with the Naval Research Laboratory, Code 6570, Washington, DC 20375-5000.

IEEE Log Number 8719141. over 30 3-dB couplers fabricated using the modified process include: less than 1.0-dB average insertion loss, controllable splitting ratio, and average polarization extinction between 15 and $20 \mathrm{~dB}$.

\section{COUPler Operation}

In this section, we discuss two particular aspects that impact performance parameters achievable for polarization preserving couplers fabricated from high birefringent fibers. Specifically, we address the impact of the fiber design and the BTF process on the achievable coupler insertion loss, splitting ratio control, and polarization extinction.

\section{A. Insertion Loss}

In our work we used Hitachi stress-induced birefringent fiber [6]. Fig. 1 illustrates the major features of the geornetry and refractive index distribution of this fiber. Typically, the core and outer diameters of the fiber are 5 and $125 \mu \mathrm{m}$, respectively, while the diameters of the major and minor axes of the stress inducing elliptic region are 60 and $30 \mu \mathrm{m}$, respectively. Also, the refractive index of the stress-inducing region is lower than that of the core and the inner and outer cladding regions. This condition leads to the outer cladding acting as a highly multimoded waveguide coaxially located with the single-mode waveguide formed by the core. In practice, however, one rarely interacts with the coaxial waveguide modes or these modes are usually stripped off by the high index jacket material.

For the case of a tapered coupler, however, at some point within the tapering process the evanescent tail of the fundamental mode extends to the point that it overlaps with the coaxial waveguide modes and at this point it can coherently couple to one or more of the modes supported by that waveguide. When exact phase matching occurs over an appropriate length, all the optical power carried by the fundamental mode can be transferred to the coaxial mode. As the tapering process proceeds phase matching to that mode is destroyed and the optical power returns to the fundamental mode. This coherent coupling can continue with subsequent coaxial modes as the tapering continues until the fundamental mode field extends to the fiber outer boundary. In Fig. 2 the optical transmission of a single Hitachi fiber during tapering is illustrated. The fiber in Fig. 2(a) was pre-etched at room temperature for 

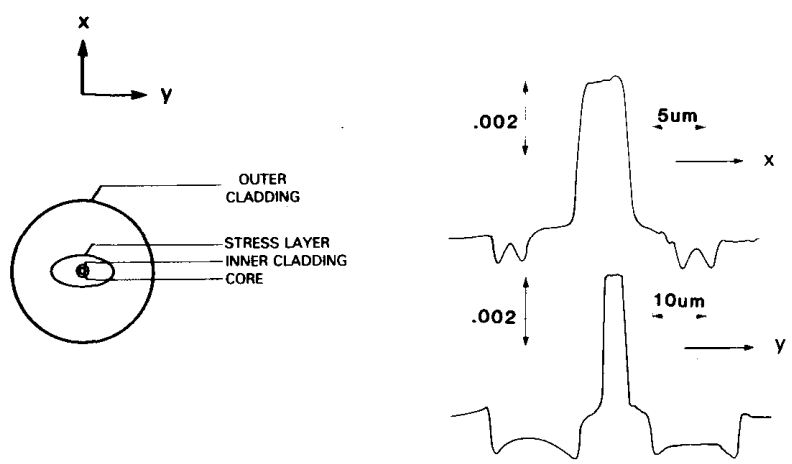

Fig. 1. Refractive index profile for standard high birefringence Hitachi fiber.

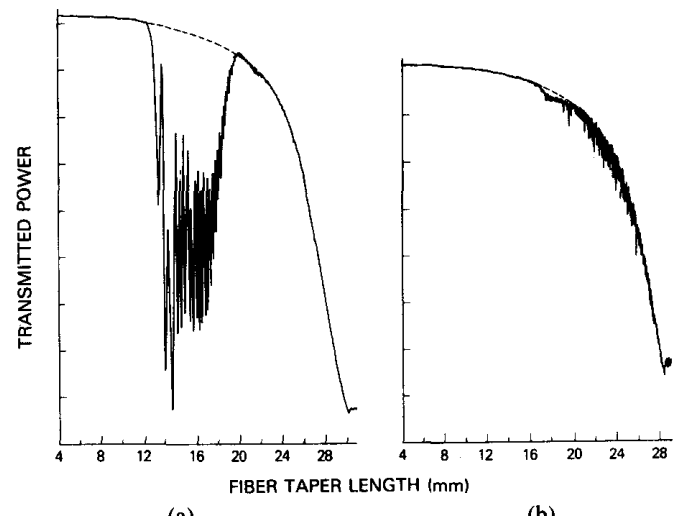

(a)

(b)

Fig. 2. Fundamental mode transmission for a single tapered Hitachi fiber: (a) 5-min pre-etch, (b) 15-min pre-etch.

five min to a final diameter approaching $110 \mu \mathrm{m}$ in $1: 1$ $\mathrm{HF}: \mathrm{H}_{2} \mathrm{O}$ prior to tapering. Note that up to near 12-mm fiber elongation no optical power loss or coupling to coaxial modes is observed and near $14 \mathrm{~mm}$ over 90 percent of the power in the core has been transferred to one or more coaxial modes. A technique commonly used to reduce this effect is to chemically pre-etch the optical fiber in order to reduce its initial outer diameter and thus reduce the mode volume of the outer coaxial waveguide. The result of pre-etching the fiber by $15 \mathrm{~min}$ to a final diameter approaching $87 \mu \mathrm{m}$ is shown in Fig. 2(b). In this result less than 10-percent power loss is observed even for a taper length approaching $20 \mathrm{~mm}$.

The increase in transmission loss beyond $20 \mathrm{~mm}$ for the 5- and 15-min pre-etched tapers is due to power transfer between the fundamental $\mathrm{HE}_{11}$ mode of the fiber and higher order modes of the fiber in the tapered regions of the fiber [7]. This mode coupling is caused by the geometric transition represented by the tapers and is irreversible, i.e., power transferred out of the $\mathrm{HE}_{11}$ mode in the input taper is not recaptured by the $\mathrm{HE}_{11}$ mode in the output taper. As the fiber is tapered the fundamental $\mathrm{HE}_{11}$ mode field expands until it reaches the fiber air-glass outer boundary. We have been able to show [8] that the onset of this taper transmission loss coincides with cutoff of the fundamental mode due to the taper slope exceeding a critical value, using a simple ray model in the regime before the expanding mode field reaches the fiber outer boundary. Fig. 2(a) and (b) illustrates this process for taper lengths greater than $20 \mathrm{~mm}$. Useful tapers longer than 20 $\mathrm{mm}$ can be fabricated by suitably adjusting the heatingelement length for a given initial diameter fiber [9].

During the coupler fabrication process it is important to be able to minimize the insertion loss and to control the splitting ratio of the coupler. A particularly effective method for minimizing the insertion loss is to pre-etch the fibers. In addition to allowing the fabrication of tapers with smaller taper slopes, pre-etching the fibers also facilitates the coupling process by requiring less tapering since the fiber cores are initially closer to one another. Also, to control the splitting ratio it is important that the coupling process be between the fundamental modes of the tapered waveguides, rather than through an intermediary higher-order coaxial mode. Fig. 3(a) and (b) shows the transmitted power during coupler fabrication for the case where the fibers were un-etched and where the fibers were pre-etched for $10 \mathrm{~min}$, respectively. The solid and broken curves correspond to the transmitted power in the main and tap fiber, respectively. The displacement between the two curves at the point where $3-\mathrm{dB}$ coupling occurs is an artifact of the two-channel chart recorder used to record the event. Note that for the case where the fibers were un-etched, (Fig. 3(a)), the final insertion loss was somewhat high, approximately $1.3 \mathrm{~dB}$. Although in this case there is substantial coupling to the coaxial waveguide modes in the 12- to 22-mm taper length region, this coupling ends as the coupling between the fundamental modes of the fibers begins, so it does not interfere with the coupling process of interest. For the case where the fibers were pre-etched by $10 \mathrm{~min}$ (Fig. 3(b)), 3-dB coupling is obtained with less than $0.5-\mathrm{dB}$ total power loss throughout the entire tapering process. In this case, since the mode volume of the coaxial waveguide is greatly reduced by pre-etching the fiber, very little coupling to coaxial waveguide modes was noticed in the $12-$ to $18-\mathrm{mm}$ region. Beyond $18 \mathrm{~mm}$, Fig. 3(b) illustrates the splitting ratio and insertion loss control achievable by the use of a microprocessor to control the tapering process. 

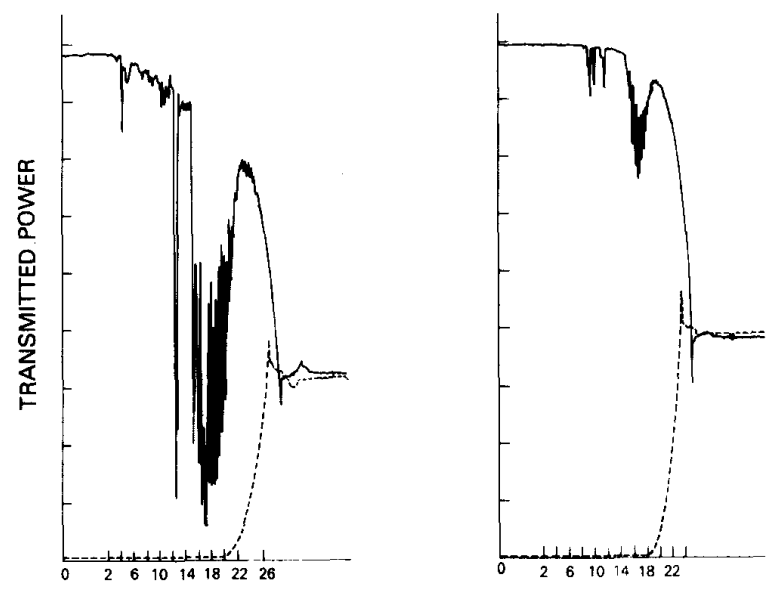

(a)

FIBER TAPER LENGTH $(\mathrm{mm})$

(b)

Fig. 3. Fundamental mode transmission for two fiber coupler: (a) fiber unetched, (b) fibers etched $10 \mathrm{~min}$. The solid line applies to the main fiber and the dashed line applies to the tap fiber.

\section{B. Polarization Extinction}

In an earlier paper [2] we reported that we had fabricated a number of polarization preserving couplers without aligning the birefringent axes of the fibers within the coupler. We explained the performance of these couplers with a model that assumed that the inherent birefringence of the fibers remained unchanged during the coupler fabrication process, i.e., radial pre-etching, tapering, and fusing of the fibers.

Although a number of the earlier couplers had very good polarization extinction, more recent efforts aimed at fabricating large numbers of these couplers did not demonstrate the fabricational reproducibility and yield desired. These results led us to investigate in detail the effect of each fabrication process step on the inherent birefringence of the Hitachi polarization preserving fiber. In [10] experimental measurements have shown a very significant reduction of the inherent birefringence of the Hitachi fiber due to both radial pre-etching and tapering of the fiber. In order to compensate for this reduced fiber birefringence, we have modified our original BTF approach by introducing a process step capable of accurate in-line alignment of the birefringent axes of the fibers within the coupling region. This modification has allowed substantially improved reproducibility in the fabrication of high-performance polarization preserving couplers.

The dependence of the polarization extinction for a 3$\mathrm{dB}$ coupler on the birefringent axis misalignment and the fiber birefringence in the coupling region is readily obtained from coupled mode theory. This problem has been dealt with in detail in [11] and the equations necessary for calculating the polarization extinction are given in that paper. The problem is complicated in that the $3-\mathrm{dB}$ coupling length becomes a function of the birefringence and the misalignment angle. Since we are chiefly concerned with reduced birefringence in the coupler we can derive an ap- proximate expression for polarization extinction by ignoring the birefringence dependence of the 3-dB coupling length and by treating each polarization independently. This approximate expression will be useful for low birefringence and for unidirectional power transfer (fiber 1 to fiber 2). We define $\theta$ to be the misalignment angle between the birefringence axes $(a, b)$ of the fibers. For an input $E_{\text {in }}$ on axis $a$ of fiber 1 , the inputs parallel to the axes of fiber 2 are $E_{\text {in }} \cos \theta$ (along $a$ ) and $E_{\text {in }} \sin \theta$ (along $b$ ). Using the coupled mode theory with an isotropic coupling $C$, the outputs of fiber 2 are

$$
\begin{aligned}
& P_{2 a}=E_{\text {in }}^{2} \cos ^{2} \theta \sin ^{2}(C L) \\
& P_{2 b}=E_{\text {in }}^{2} F^{2} \sin ^{2} \theta \sin ^{2}\left(\frac{C L}{F}\right)
\end{aligned}
$$

where

$$
F=\frac{1}{\left[1+\left(\frac{\Delta \beta_{a b}}{2 C}\right)^{2}\right]^{1 / 2}}
$$

For a $3-\mathrm{dB}$ interaction length $L$ we have $C L=\pi / 4 . L_{p}$ $=2 \pi / \Delta \beta_{a b}$ is the modified beat-length of the fibers within the coupling region. Then the polarization extinction is given by $\eta \simeq-10 \log \left(P_{2 b} / P_{2 a}\right)$ or

$\eta \simeq-10 \log \left(\frac{2 \tan ^{2} \theta \sin ^{2}\left\{\frac{\pi}{4}\left[1+\left(\frac{4 L}{L_{p}}\right)^{2}\right]^{1 / 2}\right\}}{\left[1+\left(\frac{4 L}{L_{p}}\right)^{2}\right]}\right)$

The calculated polarization extinction for a 3-dB coupler versus the angular misalignment and the ratio $L / L_{p}$ is given in Fig. 4 both for the approximate expression of (2) and for the more exact calculation of [11]. For $L / L_{p} \leqslant$ 0.4 , the calculations are nearly identical.

Note that for a typical case of $L=10 \mathrm{~mm}$, determined from the furnace filament size, 8-10 $\mu \mathrm{m}$ fiber diameter within the coupling region, and $L_{p} \gg 40 \mathrm{~mm}$ (obtained by extrapolating the results obtained in [10] for a fiber with an initial beat-length of $L_{p}=3.7 \mathrm{~mm}$ and pre-etched and tapered in a manner similar to that during coupler fabrication), the birefringent axis misalignment allowed for 15- and 20-dB polarization extinction is 11 and 6 degrees, respectively. Also note that as the ratio of $L / L_{p}$ increases, that is, more of the initial birefringence of the fiber is retained during processing, the requirement for axis alignment is significantly reduced.

Moreover, since the initial birefringence of the fibers is greatly reduced during the fabrication process, the achievable polarization extinction of BFT couplers might also be affected by the form birefringence defined by the noncircular coupler geometry. The actual geometry of our BTF couplers closely approximates that of two touching cylinders, or a "dumbbell" shape, at least within the cou- 




Fig. 4. Polarization extinction ratio as a function of misalignment angle for various values of $L / L_{p}$. Both the approximate result from (2) and the more exact analysis of [11] are shown.

pling region. If we assume that within the fused region the optical modes are those supported by the structure defined by the two touching fibers in air, we can calculate the form birefringence of the dumbbell-shaped composite waveguide using equations given by Snyder and Love [12].

The coupler has symmetric and antisymmetric normal modes with propagation constants $\beta^{s}$ and $\beta^{a}$. One can define birefringences for each of these modes by $\Delta \beta_{x y}^{s}=$ $\beta_{x}^{s}-\beta_{y}^{s}$ and $\Delta \beta_{x y}^{a}=\beta_{x}^{a}-\beta_{y}^{a}$, where $x$ and $y$ are the axes defined by the two touching fibers. Using the equations given in [12] one can show that $\left|\Delta \beta_{x y}^{s}\right|=\left|\Delta \beta_{x y}^{a}\right|=$ $\left|\Delta \beta_{x y}\right|$ and that

$$
\left|\Delta \beta_{x y}\right|=\left(\frac{2}{\pi}\right)^{1 / 2} \frac{\Delta^{3 / 2} U^{2}}{\rho V^{7 / 2}}
$$

where $\rho$ is the reduced radius of each fiber after etching and tapering. Here $\Delta$ and $V$ have the usual definitions with air as the cladding material $\left(n_{\text {clad }}=1.0\right)$.

$$
\begin{aligned}
& \Delta=\frac{n^{2}-1}{2 n^{2}} \\
& V=\frac{2 \pi \rho n}{\lambda}(2 \Delta)^{1 / 2} .
\end{aligned}
$$

In (4) $n$ is the fiber refractive index and $\lambda=0.83 \mu \mathrm{m}$ is the optical wavelength. For $V$ large we approximate $U$ by $U_{\infty}=2.405$. Equation (3) has also been derived in [13].

For $n=1.46$ we calculate the beat length due to the form birefringence or coupler shape as

$$
L_{s}=\frac{2 \pi}{\left|\Delta \beta_{x y}\right|}=1.710^{4} \rho^{9 / 2} .
$$

For the actual case of a 3-dB coupler, $\rho=4$ to $5 \mu \mathrm{m}$ [14] and the corresponding beat length is $L_{s}=9$ to $23 \mathrm{~m}$. Thus, the form birefringence due to the coupler shape is presumably still smaller than our reduced fiber birefringence in the coupler $\left(L_{p} \gg 40 \mathrm{~mm}\right)$.

A remaining question to consider is whether when aligning the fiber birefringence axes, preparatory to making the coupler, it is also necessary to align the fiber birefringence axes with the form birefringence axes. We can estimate the worst case by neglecting the fiber birefringence in the coupling region, and assume the fiber birefringence axes outside the coupling region to be aligned to each other but at a $45^{\circ}$ angle to the $x, y$ axes defined by the form birefringence ( $a, b$ axes rotated $45^{\circ}$ from $x, y$ axes). The input fields are then $E_{x}=E_{y}=$ $E_{\text {in }} / \sqrt{2}$. We assume unequal coupling coefficients $C_{x}$ and $C_{y}$ and $\Delta \beta_{a b}=0$. The coupled fields are

$$
\begin{aligned}
& E_{2 x}=\frac{E_{\text {in }}}{\sqrt{2}} \sin C_{x} L \\
& E_{2 y}=\frac{E_{\text {in }}}{\sqrt{2}} \sin C_{y} L
\end{aligned}
$$

and the output powers aligned along the output fiber's axes are

$$
\begin{aligned}
& P_{2 a}=\frac{E_{\text {in }}^{2}}{2} \cos ^{2}\left[\left(C_{x}-C_{y}\right) \frac{L}{2}\right] \\
& P_{2 b}=\frac{E_{\text {in }}^{2}}{2} \sin ^{2}\left[\left(C_{x}-C_{y}\right) \frac{L}{2}\right]
\end{aligned}
$$

where we have used $C=\left(C_{x}+C_{y}\right) / 2$ and for a $3-\mathrm{dB}$ coupler, $C L=\pi / 4$. The polarization extinction is then given by

$$
\eta=-10 \log \left[\tan ^{2}\left(\frac{C_{x}-C_{y}}{2}\right) L\right]
$$

From [12] or [13] we have

$$
\left|\frac{C_{x}-C_{y}}{C}\right|=\frac{\Delta}{V}
$$

so (8) becomes

$$
\eta=-10 \log \left[\tan ^{2}\left(\frac{\pi \Delta}{8 V}\right)\right]
$$

which remains less than $-50 \mathrm{~dB}$ for $\rho=4$ to $5 \mu \mathrm{m}$.

Therefore, it appears that shape birefringence effects are significantly smaller than those due to reduced fiber birefringence. As a result we do not have the additional requirement that the birefringent axis of the dumbbellshaped waveguide must be aligned with the birefringent axes of the coupler input-output fiber leads. In practice 
for one of the alignment techniques discussed below (spot alignment technique), we automatically obtained alignment between the shape birefringence of the coupler and that of the coupler leads. However, we have not been able to measure any benefit due to this alignment, which is consistent with the theoretical modeling given above.

\section{Coupler Fabrication}

In this section we discuss those aspects of the coupler fabrication technique that are substantially different from those described in [2]. In particular, owing to the substantial reduction of the inherent fiber birefringence during radial chemical pre-etching and/or thermal tapering of the fibers, we have modified our process to include an inline method to identify and align the birefringent axes of the fibers comprising the coupler. Two distinct methods were developed and are outlined below. Additionally, we describe the packaging method used to support and protect the fragile tapered fibers.

Common to both coupler fabrication methods is the technique and apparatus used to inject light from a superluminescent diode (SLD) into the input end of one of the high birefringent fibers. The injected light is first plane polarized and its azimuth is aligned with either the fast or slow axis of the fiber.

\section{A. End-To-End Alignment Method}

The first alignment approach will be referred to as the "end-to-end" method. With it we project the axis alignment at a reference point to another point along the length of the fiber. This method assumes that once either birefringent axis orientation, at the output end of the fiber, is identified and fixed relative to an arbitrary laboratory reference orientation, the axis alignment is maintained over a sufficient fiber length suitable for coupler fabrication. The useful fiber length is limited to about $40 \mathrm{~cm}$ by unavoidable axis rotation internal to the fiber averaging approximately 1.0 degree per centimeter [15], and by twists of the fiber and its axis within the polymer jacket that are added during setup and can be expected to be equal in magnitude to the axis rotation internal to the fiber.

Fig. 5 illustrates the end-to-end alignment method and the apparatus used to implement it. First a suitable length of the output end of the fiber is fed through and affixed to the rotation mount. Then the polarization extinction ratio at the output end of the fiber is measured and the fiber is rotated via the rotation mount until the fiber axis orientation matches a laboratory reference orientation. Since the maximum polarization extinction ratio is extremely sensitive to the analyzer azimuth angle, either birefringent axis of the fiber can be identified and aligned to an accuracy of less than one degree.

Once the fiber has been aligned to the laboratory frame, its orientation is maintained by affixing the fiber to microscope cover-glass flats located on either side of the mounting stages. Some care is required during this step of the process in order not to introduce additional rotation or bending of the fiber. Both the main and the tap fiber

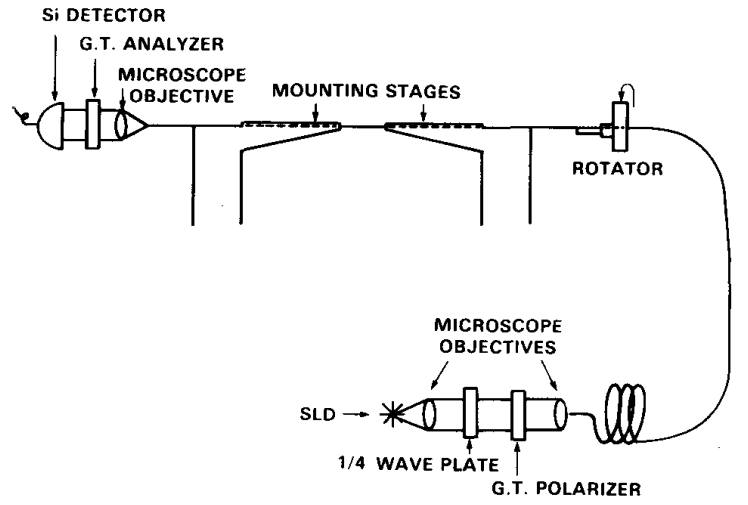

Fig. 5. Experimental arrangement for the end-to-end alignment technique.

leads are sequentially aligned and affixed in the same manner. The fiber length for both the main and tap fibers, from the output end facet to the center of the mounting stages, is held constant to within a few millimeters in order to compensate for length-dependent variations of the axis rotation internal to the fiber.

The coupler is fabricated by first placing the exposed sections of both pre-aligned fibers in contact with each other. This is done by repeatedly overlapping one fiber over the other several times while taking care not to introduce any permanent rotation/twist of the fiber or its jacket, then radially pre-etching the fibers in a suitable HF acid solution and heating and tapering the fibers until the required power splitting is obtained.

\section{B. Spot Alignment Method}

The second alignment approach will be referred to as the "spot alignment" method. Here, the axis orientation is identified only within a very small region of the fiber, less than $1 \mathrm{~cm}$, and it is assumed that the orientation is maintained over a length sufficient for coupler fabrication. This method assumes the coincidence of the major and minor axes of the elliptical stress region of the Hitachi fiber and its birefringent axes. Further, one requires the existence of a differential birefringence within the stress region and the fiber outer cladding. Then, by transversely illuminating the fiber, between crossed polarizers, one can visualize the rotationally asymmetric birefringence of the elliptic region. Thus, as the fiber is axially rotated its major and minor axes are readily identified [3]. Fig. 6(a) illustrates the spot-alignment method and the apparatus used to implement it. Here too, a suitable length of fiber is first inserted and affixed to the rotation mount. Then the fiber is placed in an index matched cell that is co-aligned with the optical arrangement containing polarized bottom illumination, cross-polarized viewing, and $200 \times$ optical magnification. Once the desired axis and its orientation have been established at a point on the fiber, by visually observing either of the images shown in Fig. 6(b) or (c), the fiber orientation is secured much in the same manner as described in the "end-to-end" method. With this method, we are able to identify and affix the fiber axes at 




(a)
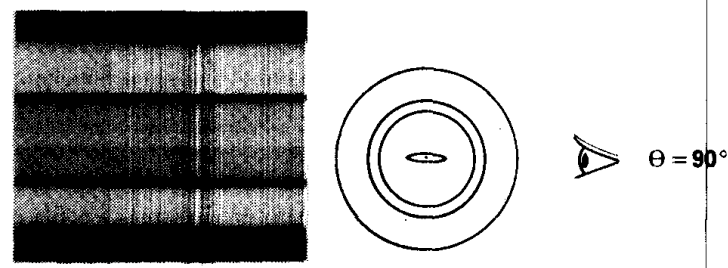

(b)
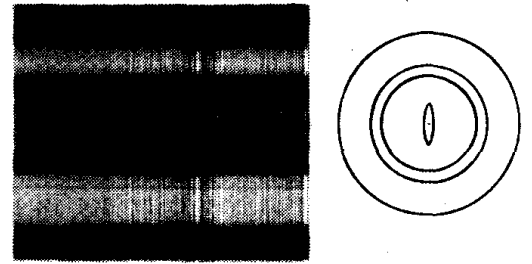

(c)

Fig. 6. Experimental arrangement for the spot-aligning technique: (a) ap paratus setup, (b) transverse fiber view for $\theta=90^{\circ}$, (c) transverse fiber view for $\theta=0^{\circ}$.

any location along the fiber and, so long as one is able to freely rotate the fiber, the coupler leads can be of any length. Once both fibers, the main and the tap, have been processed in this manner, the coupler fabrication steps are identical to those described for the "end-to-end" method.

\section{Packaging Approach}

Once the tapering process is completed and the desired splitting ratio is obtained, a mechanical package is added to the fragile biconically tapered and fused fibers. The package used with our devices is illustrated in Fig. 7. It consists of two fused silica glass segments: a half cylinder approximately $4 \mathrm{~mm}$ in outer diameter and 2.5-mm inner diameter and a flat strip 4-mm wide and $1-\mathrm{mm}$ thick. In applying the package, we first bring the half cylinder from below the fibers, we than apply a fast curing two-part epoxy to both ends of the coupler well beyond the coupling region. Sufficient epoxy is applied so that sections from both the bare and jacketed fibers are bonded to the glass half cylinder. Before the epoxy is completely cured the flat segment is added to complete the fiber enclosure.

\section{Coupler Characterization and Results}

The use of broad spectral bandwidth sources, such as tungsten lamps or super-luminescent diodes, is now the standard method used to measure the extinction ratio of

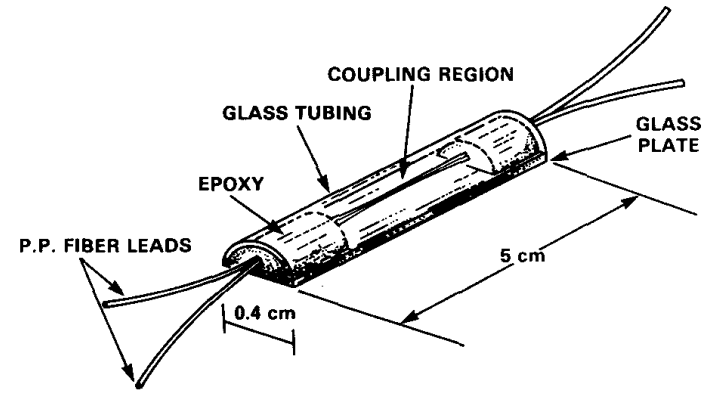

Fig. 7. Coupler packaging arrangement.

polarization preserving fibers and couplers [16]. We used an SLD operating at $0.83-\mu \mathrm{m}$ center wavelength during the fabrication and characterization of each coupler.

We measured the coupler insertion loss, splitting ratio, and the polarization extinction ratio for both coupler output fibers. Alignment of the plane polarized light from the SLD to the birefringent axis of the coupler input fiber is reproducibly done to an accuracy of better than 1 degree. Table I summarizes the results for the best 10 out of 19 couplers made using the end-to-end alignment technique. Note that the polarization extinction for the tap fiber is usually higher than that for the main fiber. We believe that this is due to the fact that once the tap fiber is aligned, it remains undisturbed throughout the entire coupler fabrication process. In contrast, once the main fiber has been aligned it must be overlaid several times over the tap fiber and therefore its birefringent axis alignment can be altered during this operation. The values given in parentheses are estimates for the axis misalignment and are obtained from (2) by assuming that $L / L_{p}<0.1$ and that axis misalignment is the only factor degrading the extinction ratio. That is to say, the remaining birefringence of the fibers in the coupling region is negligible such that $L_{p} \gg 40 \mathrm{~mm}$. Note that although for this alignment approach we can align the axes to better than one degree at the fiber ends, the other steps of the process and the internal axis rotation [15] apparently can introduce significant axis misalignment. Alternatively, other unmodeled factors exist that influence the extinction ratio. The fact that the extinction ratios for the main and tap fibers can be significantly different implies that the latter situation exists to some extent.

In Table II we summarize the results for the 10 out of 12 couplers made using the spot alignment technique. In this case the main fiber often had a higher polarization extinction than the tap. Since we utilized a similar setup process to that used with the end-to-end alignment approach, we believe that this result was due to a combination of effects due to the fiber setup process and the accuracy of the axis alignment approach used. We feel that our attempts to avoid bending or twisting the fibers during the setup process sometimes fortuitously compensated for errors of plus or minus two or three degrees introduced during the axis alignment. Again the values in parentheses represent an estimate for the angular mis- 
TABLE I

Performance Results for End-To-End Alignment Technique

\begin{tabular}{c|c|c|c|c}
\hline \hline & \multirow{2}{*}{$\begin{array}{c}\text { LOSS } \\
\text { (dB) }\end{array}$} & \multirow{2}{*}{$\begin{array}{c}\text { MAIN } \\
\text { (\%) }\end{array}$} & \multicolumn{2}{|c}{ EXTINCTION RATIO (db) } \\
\cline { 5 - 6 } & & MAIN & TAP \\
\hline A & 0.50 & 65 & 19.5 & 23.5 \\
B & 0.73 & 58 & 19.5 & 19.8 \\
C & 0.74 & 54 & 17.1 & 23.4 \\
D & 0.62 & 55 & 15.9 & 29.3 \\
E & 0.64 & 57 & 16.4 & 26.9 \\
F & 0.82 & 44 & 16.8 & 26.1 \\
G & 0.61 & 54 & 23.8 & 21.7 \\
H & 0.67 & 55 & 23.3 & 21.9 \\
I & 0.60 & 56 & 16.8 & 25.3 \\
J & 0.64 & 55 & 18.8 & 19.8 \\
\hline AVERAGE VALUES: & & & \\
N = 10 & 0.66 & 55 & $18.0\left(7.2^{\circ}\right)$ & $22.6\left(4.2^{\circ}\right)$ \\
N = 19 & 0.69 & 54 & $16.7\left(8.3^{\circ}\right)$ & $18.1\left(7.1^{\circ}\right)$ \\
\hline
\end{tabular}

TABLE II

Performance Results for the Spot Alignment Trehnique

\begin{tabular}{|c|c|c|c|c|}
\hline & \multirow{2}{*}{$\begin{array}{l}\text { LOSS } \\
\text { (dB) }\end{array}$} & \multirow{2}{*}{$\begin{array}{l}\text { MAIN } \\
(\%)\end{array}$} & \multicolumn{2}{|c|}{ EXTINCTION RATIO (dB) } \\
\hline & & & MAIN & IAP \\
\hline A & 0.70 & 57 & 17.0 & 13.0 \\
\hline B & 0.45 & 51 & 17.1 & 13.0 \\
\hline c & 0.54 & 44 & 13.1 & 20.0 \\
\hline D & 0.49 & 52 & 15.0 & 16.1 \\
\hline E & 0.45 & 57 & 14.1 & 17.1 \\
\hline $\mathbf{F}$ & 0.48 & 56 & 23.7 & 14.5 \\
\hline G & 0.85 & 55 & 29.2 & 16.8 \\
\hline H & 0.42 & 51 & 18.4 & 14.0 \\
\hline 1 & 0.63 & 55 & 25.0 & 14.3 \\
\hline$J$ & 0.64 & 55 & 22.4 & 15.1 \\
\hline \multicolumn{5}{|c|}{ AVERAGE VALUES: } \\
\hline$N=10$ & 0.57 & 53 & $17.6\left(7.51^{\circ}\right)$ & $15.4\left(9.64^{\circ}\right)$ \\
\hline$N=12$ & 0.59 & $! 3$ & $16.5\left(8.52^{\circ}\right)$ & $15.4\left(9.64^{\circ}\right)$ \\
\hline
\end{tabular}

alignment assuming that $L / L_{p} \ll 0.1$ and that axis misalignment is the only factor degrading the extinction ratio.

The results summarized in Tables I and II demonstrate conclusively that both birefringent axis alignment techniques, although limited by axis rotation within the fiber and unavoidable fiber twist during setup, provide the required alignment accuracy to allow the fabrication of polarization preserving couplers with low loss, controllable splitting ratio, and polarization extinction of 15 to $20 \mathrm{~dB}$. Our experience was that the fabrication yield for these devices approached 50 percent for both alignment techniques, considering only the devices that reached the measuring stage. Additional device performance improvements should be possible by reducing or eliminating the internal axis rotation found in most high birefringent fibers and by further refinements of the alignment methods outlined here.

\section{Conclusions}

We have outlined two loss mechanisms that, when uncontrolled, can lead to high insertion loss during the fabrication of BTF couplers using stress-induced Hitachi high-birefringent fiber. We have also quantified the birefringent axis alignment requirements for high-performance polarization preserving couplers by taking into ac- count the significant reduction of the inherent fiber birefringence due to radial pre-etching and tapering of $\mathrm{Hi}$ tachi high-birefringence fiber. Additionally, we have quantified the form birefringence due to coupler shape and conclude that its contribution to the polarization performance of our couplers is negligible. Further, we have outlined two in-line techniques for identifying and aligning the birefringent axes of high-birefringent fiber within the coupling region prior to coupler fabrication. We have also demonstrated that both methods are useful for the fabrication of high-performance polarization preserving couplers that are: low loss, have controllable splitting ratio, and offer a polarization extinction of 15 to $20 \mathrm{~dB}$. Finally, by fabricating over 19 couplers, we have demonstrated that our modified BTF process with end-to-end axis alignment offers close to 50-percent yield for high-performance $20-\mathrm{dB}$ mean polarization extinction, polarization preserving couplers. The fundamental performance, in terms of polarization extinction, for BTF polarization preserving couplers is coupled to the significant loss of birefringence [10] due to etching and tapering of the fiber, and the inherent birefringent axis rotation [15] in the fiber. However, the devices we have fabricated are suitable for most applications where additional external perturbations, e.g., fiber bending or twisting within the coupling region, leading to significant polarization coupling, are not present. Therefore, we believe that these results should significantly impact the continuing development of communication and sensor systems that use high-birefringent fiber.

\section{ACKNOWLEDGMENT}

The authors thank R. P. Moeller for obtaining the index profile used in Fig. 1.

\section{REFERENCES}

[1] M. Kawachi, B. S. Kawasaki, K. O. Hill, and T. Edahiro, "Fabrication of single polarization single mode fibre couplers," Electron. Lett., vol. 18, pp. 962-964, 1982.

[2] C. A. Villarruel, M. Abebe, and W. K. Burns, "Polarization preserving single-mode fiber coupler," Electron. Lett., vol. 19, pp. 17$18,1983$.

[3] H. Uetsuka, Y. Fujii, and Y. Mutsuhashi, "Tunable directional coupler using single polarization fiber,'" presented at the Integrated Optics Optical Communications Conf., Tokyo, paper 29A5-2PD, 1983.

[4] R. B. Dyott and J. Bello, "Polarization holding directional couple made from elliptically cored fibre having a D section,', Electron. Lett., vol. 19, pp. 601-602, 1983.

[5] W. Pleibel, R. H. Stolen, and S. C. Rashleigh, "Polarization preserving coupler with self aligning birefringent fibers," Electron. Lett. vol. 19, pp. 19-20, 1983.

[6] T. Katsuyama, H. Matsumura, and T. Suganuma, "Low-loss single polarization fibre," Electron. Lett., vol. 17, pp. 473-474, 1981.

[7] S. Lacroix, R. Bourbonnais, F. Gonthier, and J. Bures, "Tapered monomode optical fibers: understanding large power transfer,"' Appl. Opt., vol. 25, pp. 4421-4425, 1986.

[8] W. K. Burns, M. Abebe, C. A. Villarruel, and R. P. Moeller, "Loss mechanisms in single mode fiber tapers," J. Lightwave Tech., vol. LT-4, pp. 608-613, 1986.

[9] W. K. Burns, M. Abebe, and C. A. Villarruel, "Parabolic model for shape of fiber taper,"' Appl. Opt., vol. 24, pp. 2753-2755, 1985.

[10] C. A. Villarruel, M. J. Marrone, N. J. Frigo, and A. Dandridge, "Birefringence in etched and tapered polarization preserving fibers," presented at the 6th Int. Conf. Integrated Optics Optical Fiber Communications, Reno, 1987. 
[11] C.-L. Chen and W. K. Burns, "Polarization characteristics of singlemode fiber couplers," IEEE J. Quantum Electron., vol. QE-18, pp. $1589-1600,1982$

[12] A. W. Snyder and J. D. Love, Optical Waveguide Theory. New York: Chapman and Hall, 1983, ch. 18.

[13] A. Ankiewicz, A. W. Snyder, and X.-H. Zheng, "Coupling between parallel optical fiber cores-critical examination," J. Lightwave Tech., vol. LT-4, pp. 1317-1323, 1986.

[14] W. K. Burns and M. Abebe, "Coupling model for fused fiber couplers with parabolic taper shape," Appl. Opt., vol. 26, pp. 4190$4192,1987$.

[15] M. J. Marrone, C. A. Villarruel, N. J. Frigo, and A. Dandridge, "Internal rotation of the birefringence axes in polarization holding fibers," Opt. Lett., vol. 12, pp. 60-62, 1987.

[16] S. C. Rashleigh, W. K. Burns, R. P. Moeller, and R. Ulrich, "Polarization holding in birefringent single mode fibers," Opt. Lett., vol. 7, pp. $40-42,1982$

M. Abebe, photograph and biography not available at the time of publication.

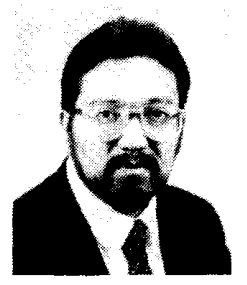

Carl A. Villarruel was born in Quito, Ecuador, in January 1948. He received the B.E. and M.E. degrees in electrical engineering from The City College of New York, New York, NY, in 1971 and 1973 , respectively.

Since 1975 he has been a Research Engineer in the Optical Sciences Division at the Naval Research Laboratory, Washington, DC. His research interests include multimode and single-mode fiber optic coupler technology, and their application to communications and sensor systems. He has au- thored and coauthored over 25 journal publications and conference presentations. In 1984 he served as Conference Chairman for the SPIE Conference on Fiber Optic Couplers, Connectors and Splice Technology, Washington, DC.

Mr. Villarruel is a member of the Optical Society of America.

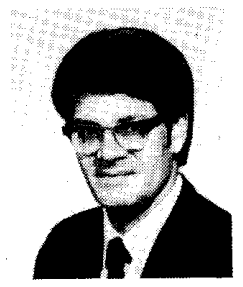

William K. Burns (M'80) was born in Philadelphia, PA, in June 1943. He received the B.S. de gree in engineering physics from Cornell University, Ithaca, NY, in 1965, and the M.S. and Ph.D. degrees in applied physics from Harvard University, Cambridge, MA, in 1967 and 1971 , respectively. His thesis research was in nonlinear optics

During 1971 he was a staff member at Arthur

D. Little, Inc., Cambridge, MA, where he contributed to various laser-related studies and surveys. Since 1972 he has been a Research Physicist at the Naval Research Laboratory, Washington, DC. He is presently Head of the Optical Waveguide Section of the Optical Techniques Branch. His research interests include integrated optics, single-mode fiber optics, and the application of single-mode technology to communications, sensors, and signal processing.

Dr. Burns received NRL's Sigma Xi award for applied science in 1985 and is a fellow of the Optical Society of America. 